\title{
Use of 2-aminoprop-1-ene-1,1,3-tricarbonitrile for the synthesis of tetrahydronaphthalene, hexahydroisoquinoline and hexahydrocinnoline derivatives with potential antitumor activities
}

\author{
RAFAT M. MOHAREB ${ }^{1,2 *}$ \\ HOSAM E. MOUSTAFA ${ }^{3}$ \\ ${ }^{1}$ October University for Modern \\ Sciences and Arts, El Wahaat Road \\ October City, A.R. Egypt \\ 2 Chemistry Department, Faculty \\ of Science, Cairo University \\ Giza, A.R. Egypt \\ 3 Applied Medical Sciences \\ Department Community College \\ in Quaryat, Al Jouf University \\ Al Jouf, Saudi Arabia
}

Accepted December 15, 2010

\begin{abstract}
The aim of the work was to synthesize heterocyclic compounds from 2-aminoprop-1-ene-1,1,3-tricarbonitrile and to study their antitumor activities. The title reagent reacted with cyclohexanone to give the ethylidene derivative 2 . The reactivity of the latter product towards different chemical reagents was studied to give tetrahydronaphthalene, hexahydroisoquinoline and hexahydrocinnoline derivatives. The newly synthesized products were screened as antitumor agents on the in vitro growth of three human tumor cell lines representing different tumor types, namely, breast adenocarcinoma (MCF-7), non-small cell lung cancer (NCI-H460) and CNS cancer (SF-268). It was found that some of these compounds showed inhibitory effects on the three cell lines, indicating their potential use in the development of oncology products.
\end{abstract}

Keywords: tetrahydronaphthalene, hexahydroisoquinoline, hexahydrocinnoline, antitumor activity

2-Aminoprop-1-ene-1,1,3-tricarbonitrile has attracted a great deal of interest due to its wide applications in the field of pharmaceuticals (1). The title reagent had great applicability in heterocyclic synthesis since it was used for the synthesis of pyridines, pyrimidines, pyridazines, thiophenes, thiazoles and their analogs $(2,3)$. The importance of such compounds is due to their diverse pharmaceutical activities including for neurological disorders (4), as receptor antagonists (5), antidiabetics (6), tubulin inhibitors (7), kinase inhibitors (8) and anticancer agents (9). In continuation of our interest in the development of new and simple methods for the synthesis of polyfunctionally substituted heterocycles with anticipated biological activity (10), we report here the uses of the title reagent for the synthesis of new tetrahydronaphthalene, hexahydroquinoline and hexahydrocinnoline derivatives together with their antitumor evaluation.

\footnotetext{
*Correspondence; e-mail: raafat_mohareb@yahoo.com
} 
R. M. Mohareb and H. E. Moustafa: Use of 2-aminoprop-1-ene-1,1,3-tricarbonitrile for the synthesis of tetrahydronaphthalene, hexahydroisoquinoline and hexahydrocinnoline derivatives with potential antitumor activities, Acta Pharm. 61 (2011) 51-62.

\section{EXPERIMENTAL}

All melting points were determined on an electrothermal apparatus (Büchi 535, Switzerland) in an open capillary tube and were uncorrected. Elemental analyses were performed on a Yanaco CHNS Corder elemental analyzer (Japan). IR spectra $\left(v, \mathrm{~cm}^{-1}\right)$ were recorded in $\mathrm{KBr}$ pellets on a PA-9721 IR spectrophotometer (Shimadzu, Japan). ${ }^{1} \mathrm{H}$ NMR and ${ }^{13} \mathrm{C}$ NMR spectra were obtained on a Jeol $300 \mathrm{MHz}$ (Japan) spectrometer in DMSO- $d_{6}$ as solvent, using TMS as internal reference, and chemical shifts $(\delta)$ are expressed in ppm. Mass spectra were recorded on Kratos (75 eV) MS equipment (Germany).

Synthetic pathways are presented in Schemes 1-3 and physicochemical and spectral data of the synthesized compounds are given in Tables I and II. 2-Amino-3-cyclohexylideneprop-1-ene-1,1,3-tricarbonitrile (1) was synthesized according to the literature procedure (11). Compounds $\mathbf{4} \mathbf{a}, \mathbf{b}$ and $\mathbf{6} \mathbf{a}, \mathbf{b}$ were formed through non-isolable intermediates $3 a, b$ and $5 a, b$, respectively.

\section{Syntheses}

2-Amino-3-(2-benzylidenecyclohexylidene)prop-1-ene-1,1,3-tricarbonitrile (2). - To a solution of compound $1(0.212 \mathrm{~g}, 1 \mathrm{mmol})$ in ethanol $(40 \mathrm{~mL})$, piperidine $(0.5 \mathrm{~mL})$ and benzaldehyde $(0.106 \mathrm{~g}, 1 \mathrm{mmol})$ were added. The reaction mixture was heated under reflux for $3 \mathrm{~h}$, then poured onto an ice/water mixture containing a few drops of hydrochloric acid. The formed solid product was collected by filtration.

3-Amino-4-(1-amino-2,2-dicyanovinyl)-1-phenyl-5,6,7,8-tetrahydro-1-phenylnaphthalene-2 -carbonitrile (4a) and ethyl 3-amino-4-(1-amino-2,2-dicyanovinyl)- 5,6,7,8-tetrahydro-1-phenylnaphthalene-2-carboxylate (4b). General procedure. - Method A. To a solution of compound 2 $(0.3 \mathrm{~g}, 1 \mathrm{mmol})$, either malononitrile $(0.066 \mathrm{~g}, 1 \mathrm{mmol})$ or ethyl cyanoacetate $(0.113 \mathrm{~g}, 1$ mmol) was added in ethanol $(40 \mathrm{~mL})$ containing triethylamine $(0.50 \mathrm{~mL})$. The reaction mixture was heated under reflux for $3 \mathrm{~h}$, then poured onto an ice/water mixture containing a few drops of hydrochloric acid. The solid product formed in each case was collected by filtration. Method B. To a solution of compound $1(0.212 \mathrm{~g}, 1 \mathrm{mmol})$ in ethanol $(40 \mathrm{~mL})$ containing a catalytic amount of triethylamine $(0.50 \mathrm{~mL})$, either $\alpha$-cyanocinnamonitrile $(0.150 \mathrm{~g}, 1 \mathrm{mmol})$ or ethyl $\alpha$-cyanocinnamate $(0.201 \mathrm{~g}, 1 \mathrm{mmol})$ was added. The reaction mixture in each case was heated under reflux for $3 \mathrm{~h}$, then neutralized by pouring onto an ice/ water mixture containing a few drops of hydrochloric acid. The solid product formed in each case was collected by filtration.

2-(Amino(2,3-diamino-1-phenyl-1,2,5,6,7,8-hexahydroisoquinolin-4-yl)methylene)malononitrile (6a) and 2-(amino(3-amino-1-phenyl-2-(phenylamino)-1,2,5,6,7,8-hexahydroisoquinolin-4-yl)methylene)-malononitrile (6b). - To a solution of compound $2(0.30 \mathrm{~g}, 1 \mathrm{mmol})$ in ethanol $(40 \mathrm{~mL})$, either hydrazine hydrate $(0.050 \mathrm{~g}, 1 \mathrm{mmol})$ or phenylhydrazine $(0.108 \mathrm{~g}, 1$ $\mathrm{mmol}$ ) was added. The reaction mixture in each case was heated under reflux for $3 \mathrm{~h}$, then poured onto an ice/water mixture containing a few drops of hydrochloric acid. The formed solid product in each case was collected by filtration.

2-Amino-3-(2-benzylidene-6-bromocyclohexylidene)prop-1-ene-1,1,3-tricarbonitrile (7). A solution of compound $2(0.30 \mathrm{~g}, 1 \mathrm{mmol})$ in glacial acetic acid $(30 \mathrm{~mL})$ was warmed to 
R. M. Mohareb and H. E. Moustafa: Use of 2-aminoprop-1-ene-1,1,3-tricarbonitrile for the synthesis of tetrahydronaphthalene, hexahydroisoquinoline and hexahydrocinnoline derivatives with potential antitumor activities, Acta Pharm. 61 (2011) 51-62.

$60{ }^{\circ} \mathrm{C}$, then bromine solution $(0.16 \mathrm{~g}, 1 \mathrm{mmol})$ in acetic acid $(10 \mathrm{~mL})$ was added dropwise under continuous stirring. The reaction mixture was stirred for $1.5 \mathrm{~h}$, then poured into an ice/water mixture and the formed solid product was collected by filtration.

4-(2-Benzylidenecyclohexylidene)(cyano)methyl)-1,2,3,6-tetrahydro-6-imino-1-phenyl-2-thioxopyrimidine-5-carbonitrile (8). - To a solution of compound $2(0.30 \mathrm{~g}, 1 \mathrm{mmol})$ in ethanol $(40 \mathrm{~mL})$ containing triethylamine $(0.50 \mathrm{~mL})$, phenylisothiocyanate $(0.135 \mathrm{~g}, 1 \mathrm{mmol})$ was added. The reaction mixture was heated under reflux for $3 \mathrm{~h}$, then poured into an ice/ water mixture containing a few drops of hydrochloric acid. The formed product was collected by filtration.

3-Amino-2-cyano-4-(2-(p-tolyl-hydrazono)-cyclohexylidene)prop-1-ene-1,1,3-tricarbonitrile (9). - To a cold solution $\left(0-5^{\circ} \mathrm{C}\right)$ of compound $\mathbf{1}(0.212 \mathrm{~g}, 1 \mathrm{mmol})$ in pyridine $(15 \mathrm{~mL})$, an equimolar amount of diazotized-4-methyl-benzenediazonium chloride was gradually added under stirring. The solid product separated during stirring in an ice bath was collected by filtration and washed thoroughly with water.

Table I. Physical properties of the newly synthesized compounds

\begin{tabular}{|c|c|c|c|c|c|c|c|}
\hline \multirow{2}{*}{$\begin{array}{c}\text { Compd. } \\
\text { No. }\end{array}$} & \multirow{2}{*}{$\begin{array}{l}\text { M.p. }\left({ }^{\circ} \mathrm{C}\right) \\
\text { (solvent of } \\
\text { cryst.) }\end{array}$} & \multirow{2}{*}{$\begin{array}{c}\text { Yield } \\
(\%)\end{array}$} & \multirow{2}{*}{$\begin{array}{l}\text { Mol. formula } \\
\qquad\left(M_{\mathrm{r}}\right)\end{array}$} & \multicolumn{4}{|c|}{ Analysis calcd./found (\%) } \\
\hline & & & & $\mathrm{C}$ & $\mathrm{H}$ & $\mathrm{N}$ & $\mathrm{S}$ \\
\hline 2 & $\begin{array}{c}143-145 \\
\text { (dioxane) }\end{array}$ & 67 & $\begin{array}{c}\mathrm{C}_{19} \mathrm{H}_{16} \mathrm{~N}_{4} \\
(300.14)\end{array}$ & $\begin{array}{l}75.98 \\
75.86\end{array}$ & $\begin{array}{l}5.37 \\
5.29\end{array}$ & $\begin{array}{l}18.65 \\
18.73\end{array}$ & \\
\hline $4 a$ & $\begin{array}{c}221-224 \\
(\mathrm{EtOH})\end{array}$ & 79 & $\begin{array}{c}\mathrm{C}_{21} \mathrm{H}_{17} \mathrm{~N}_{5} \\
(339.39)\end{array}$ & $\begin{array}{l}74.32 \\
74.14\end{array}$ & $\begin{array}{l}5.05 \\
5.12\end{array}$ & $\begin{array}{l}20.63 \\
20.77\end{array}$ & \\
\hline $4 b$ & $\begin{array}{l}98-102 \\
(\mathrm{EtOH})\end{array}$ & 76 & $\begin{array}{c}\mathrm{C}_{23} \mathrm{H}_{22} \mathrm{~N}_{4} \mathrm{O}_{2} \\
(386.45)\end{array}$ & $\begin{array}{l}71.48 \\
71.29\end{array}$ & $\begin{array}{l}5.74 \\
6.00\end{array}$ & $\begin{array}{l}14.50 \\
14.76\end{array}$ & \\
\hline $6 a$ & $\begin{array}{l}192-196 \\
(\text { EtOH })\end{array}$ & 79 & $\begin{array}{c}\mathrm{C}_{19} \mathrm{H}_{20} \mathrm{~N}_{6} \\
(332.40)\end{array}$ & $\begin{array}{l}68.65 \\
68.58\end{array}$ & $\begin{array}{l}6.06 \\
6.24\end{array}$ & $\begin{array}{l}25.28 \\
25.54\end{array}$ & \\
\hline $6 b$ & $\begin{array}{c}137-140 \\
(\mathrm{EtOH})\end{array}$ & 67 & $\begin{array}{c}\mathrm{C}_{25} \mathrm{H}_{24} \mathrm{~N}_{6} \\
(408.50)\end{array}$ & $\begin{array}{l}73.51 \\
73.50\end{array}$ & $\begin{array}{l}5.92 \\
5.67\end{array}$ & $\begin{array}{l}20.57 \\
20.61\end{array}$ & \\
\hline 7 & $\begin{array}{l}210-212 \\
(\mathrm{AcOH})\end{array}$ & 77 & $\begin{array}{c}\mathrm{C}_{19} \mathrm{H}_{15} \mathrm{BrN}_{4} \\
(379.25)\end{array}$ & $\begin{array}{l}50.17 \\
49.96\end{array}$ & $\begin{array}{l}3.99 \\
4.08\end{array}$ & $\begin{array}{l}14.77 \\
14.64\end{array}$ & \\
\hline 8 & $\begin{array}{l}109-112 \\
(\mathrm{EtOH})\end{array}$ & 73 & $\begin{array}{c}\mathrm{C}_{26} \mathrm{H}_{21} \mathrm{~N}_{5} \mathrm{~S} \\
(435.54)\end{array}$ & $\begin{array}{l}71.70 \\
71.74\end{array}$ & $\begin{array}{l}4.86 \\
4.86\end{array}$ & $\begin{array}{l}16.08 \\
15.85\end{array}$ & $\begin{array}{l}7.36 \\
7.18\end{array}$ \\
\hline 9 & $\begin{array}{c}140-144 \\
(\mathrm{EtOH})\end{array}$ & 77 & $\begin{array}{c}\mathrm{C}_{19} \mathrm{H}_{18} \mathrm{~N}_{6} \\
(330.39)\end{array}$ & $\begin{array}{l}69.07 \\
69.14\end{array}$ & $\begin{array}{l}5.49 \\
5.26\end{array}$ & $\begin{array}{l}25.44 \\
25.61\end{array}$ & \\
\hline 10 & $\begin{array}{c}165-169 \\
(\mathrm{EtOH})\end{array}$ & 75 & $\begin{array}{c}\mathrm{C}_{19} \mathrm{H}_{18} \mathrm{~N}_{6} \\
(330.39)\end{array}$ & $\begin{array}{l}69.07 \\
69.13\end{array}$ & $\begin{array}{l}5.49 \\
5.64\end{array}$ & $\begin{array}{l}25.44 \\
25.67\end{array}$ & \\
\hline 11 & $\begin{array}{c}120-122 \\
\text { (dioxane) }\end{array}$ & 80 & $\begin{array}{c}\mathrm{C}_{19} \mathrm{H}_{17} \mathrm{~N}_{5} \mathrm{O} \\
(331.37)\end{array}$ & $\begin{array}{l}68.87 \\
68.62\end{array}$ & $\begin{array}{l}5.17 \\
5.08\end{array}$ & $\begin{array}{l}21.13 \\
21.25\end{array}$ & \\
\hline $12 a$ & $\begin{array}{c}\text { 143-146 } \\
\text { (dioxane) }\end{array}$ & 75 & $\begin{array}{c}\mathrm{C}_{12} \mathrm{H}_{16} \mathrm{~N}_{6} \\
(244.14)\end{array}$ & $\begin{array}{l}59.00 \\
58.81\end{array}$ & $\begin{array}{l}6.60 \\
6.54\end{array}$ & $\begin{array}{l}34.40 \\
34.24\end{array}$ & \\
\hline $12 b$ & $\begin{array}{c}\text { 165-169 } \\
\text { (dioxane) }\end{array}$ & 71 & $\begin{array}{c}\mathrm{C}_{18} \mathrm{H}_{20} \mathrm{~N}_{6} \\
(320.17)\end{array}$ & $\begin{array}{l}67.48 \\
67.35\end{array}$ & $\begin{array}{l}6.29 \\
6.30\end{array}$ & $\begin{array}{l}26.23 \\
26.48\end{array}$ & \\
\hline
\end{tabular}


R. M. Mohareb and H. E. Moustafa: Use of 2-aminoprop-1-ene-1,1,3-tricarbonitrile for the synthesis of tetrahydronaphthalene, hexahydroisoquinoline and hexahydrocinnoline derivatives with potential antitumor activities, Acta Pharm. 61 (2011) 51-62.

Table II. Spectral data of the newly synthesized compounds

\begin{tabular}{|c|c|c|c|c|}
\hline $\begin{array}{l}\text { Compd. } \\
\text { No. }\end{array}$ & $\operatorname{IR}\left(v, \mathrm{~cm}^{-1}\right)$ & $\begin{array}{c}{ }^{1} \mathrm{H} \text { NMR }\left(\mathrm{DMSO}-d_{6}\right) \\
(\delta, \mathrm{ppm})\end{array}$ & $\begin{array}{c}{ }^{13} \mathrm{C} \text { NMR }\left(\mathrm{DMSO}-d_{6}\right) \\
(\delta, \mathrm{ppm})\end{array}$ & $\begin{array}{c}\mathrm{MS} \\
\left(\mathrm{M}^{+}\right)\end{array}$ \\
\hline 2 & $\begin{array}{l}3420,3345\left(\mathrm{NH}_{2}\right) \\
3059(\mathrm{CH} \text { aromatic }) \\
2229-2204(3 \mathrm{CN}) \\
1635(\mathrm{C}=\mathrm{C})\end{array}$ & $\begin{array}{l}2.22-2.24\left(\mathrm{~m}, 4 \mathrm{H}, 2 \mathrm{CH}_{2}\right), \\
2.30-2.34\left(\mathrm{~m}, 4 \mathrm{H}, 2 \mathrm{CH}_{2}\right), \\
3.65\left(\mathrm{~s}, 2 \mathrm{H}, \mathrm{NH}_{2}\right), 6.98 \\
\left.(\mathrm{~s}, 1 \mathrm{H}, \mathrm{CH}=\mathrm{C}), 2 \mathrm{CH}_{2}\right)\end{array}$ & $\begin{array}{l}26.8,27.3,29.0,30.3 \\
\text { (cyclohexyl C), 116.0, } \\
116.9,117.3(3 \mathrm{CN}), 87.2, \\
90.5,104.2,106.2,132.1, \\
140.3(3 \mathrm{C}=\mathrm{C}), 124.1,125.3, \\
128.8,129.5 \text { (benzene C) }\end{array}$ & 300 \\
\hline $4 a$ & $\begin{array}{l}3420-3346\left(2 \mathrm{NH}_{2}\right) \\
3058(\mathrm{CH} \text { aromatic }) \\
2228-2202(3 \mathrm{CN}) \\
1630(\mathrm{C}=\mathrm{C})\end{array}$ & $\begin{array}{l}2.22-2.24\left(\mathrm{~m}, 4 \mathrm{H}, 2 \mathrm{CH}_{2}\right) \\
2.32-2.36\left(\mathrm{~m}, 4 \mathrm{H}, 2 \mathrm{CH}_{2}\right) \\
3.89-4.64\left(2 \mathrm{~s}, 4 \mathrm{H}, 2 \mathrm{NH}_{2}\right) \\
7.29-7.49\left(\mathrm{~m}, 5 \mathrm{H}, \mathrm{C}_{6} \mathrm{H}_{5}\right)\end{array}$ & $\begin{array}{l}24.2,26.8,28.3,39.2(\mathrm{cy}- \\
\text { clohexyl C), 115.8, 116.4, } \\
115.1(3 \mathrm{CN}), 54.8,88.2 \\
(\mathrm{C}=\mathrm{C}), 123.0,124.8,126.8, \\
129.0,130.3,133.7,135.9 \\
144.3 \text { (two benzene C) }\end{array}$ & 339 \\
\hline $4 b$ & $\begin{array}{l}3460-3325\left(2 \mathrm{NH}_{2}\right) \\
3060(\mathrm{CH} \text { aromatic }) \\
2882-2787\left(\mathrm{CH}_{3}, \mathrm{CH}_{2}\right) \\
2230-2218(2 \mathrm{CN}) \\
1693(\mathrm{C}=\mathrm{O})\end{array}$ & $\begin{array}{l}1.36(\mathrm{t}, 3 \mathrm{H}, J=7.22 \mathrm{~Hz}, \\
\left.\mathrm{CH}_{3}\right), 2.20-2.27(\mathrm{~m}, 4 \mathrm{H}, \\
\left.2 \mathrm{CH}_{2}\right), 2.31-2.37(\mathrm{~m}, 4 \mathrm{H}, \\
\left.2 \mathrm{CH}_{2}\right), 4.22(\mathrm{q}, 2 \mathrm{H}, J=7.22 \\
\left.\mathrm{Hz} \mathrm{CH}_{2}\right), 4.424 .45(2 \mathrm{~s}, 4 \mathrm{H}, \\
\left.2 \mathrm{NH}_{2}\right), 7.30-7.35(\mathrm{~m}, 5 \mathrm{H}, \\
\left.\mathrm{C}_{6} \mathrm{H}_{5}\right)\end{array}$ & & 386 \\
\hline $6 a$ & $\begin{array}{l}3473-3325\left(3 \mathrm{NH}_{2}\right) \\
3058(\mathrm{CH} \text { aromatic }) \\
2890-2780\left(\mathrm{CH}_{3}, \mathrm{CH}_{2}\right) \\
2229-2222(2 \mathrm{CN}) \\
1690(\mathrm{C}=\mathrm{O})\end{array}$ & $\begin{array}{l}2.34-2.46\left(\mathrm{~m}, 4 \mathrm{H}, 2 \mathrm{CH}_{2}\right), \\
2.46-2.50\left(\mathrm{~m}, 4 \mathrm{H}, 2 \mathrm{CH}_{2}\right), \\
4.25-4.62,4.80(2 \mathrm{~s}, 4 \mathrm{H}, \\
\left.2 \mathrm{NH}_{2}\right), 5.70(\mathrm{~s}, 1 \mathrm{H}, \text { pyri- } \\
\text { dine, } \mathrm{H}-6), 7.26-7.36 \\
\left(\mathrm{~m}, 5 \mathrm{H}, \mathrm{C}_{6} \mathrm{H}_{5}\right)\end{array}$ & $\begin{array}{l}23.9,25.2,28.6,38.9(\mathrm{cy}- \\
\text { clohexyl C), 116.8, } 117.3 \\
(2 \mathrm{CN}), 54.9,85.3(\mathrm{C}=\mathrm{C}) \\
122.8,126.7,129.0,130.6, \\
132.9,135.3,142.9 \text { (benzene } \\
\text { C, pyridine C) }\end{array}$ & 332 \\
\hline $6 b$ & $\begin{array}{l}3377-3370\left(2 \mathrm{NH}_{2}\right) \\
3058(\mathrm{CH} \text { aromatic }) \\
2875\left(\mathrm{CH}_{2}\right) 2225,2220 \\
(2 \mathrm{CN}), 1621(\mathrm{C}=\mathrm{C})\end{array}$ & $\begin{array}{l}2.36-2.49\left(\mathrm{~m}, 4 \mathrm{H}, 2 \mathrm{CH}_{2}\right) \\
2.50-252\left(\mathrm{~m}, 4 \mathrm{H}, 2 \mathrm{CH}_{2}\right) \\
4.62,4.78\left(2 \mathrm{~s}, 4 \mathrm{H}, 2 \mathrm{NH}_{2}\right) \\
5.68(\mathrm{~s}, 1 \mathrm{H}, \text { pyridine, } \mathrm{H}-6) \\
7.30-7.33\left(\mathrm{~m}, 10 \mathrm{H}, 2 \mathrm{C}_{6} \mathrm{H}_{5}\right) \\
7.51(\mathrm{~s}, 1 \mathrm{H}, \mathrm{NH})\end{array}$ & & 408 \\
\hline 7 & $\begin{array}{l}3443-3333\left(\mathrm{NH}_{2}\right) \\
3052(\mathrm{CH} \text { aromatic }) \\
2783\left(\mathrm{CH}_{2}\right), 2229-2215 \\
(3 \mathrm{CN}), 1646(\mathrm{C}=\mathrm{C})\end{array}$ & $\begin{array}{l}2.21-2.26\left(\mathrm{~m}, 4 \mathrm{H}, 2 \mathrm{CH}_{2}\right) \\
2.33\left(\mathrm{~m}, 2 \mathrm{H}, \mathrm{CH}_{2}\right), 3.63 \\
(\mathrm{~m}, 1 \mathrm{H}, \text { cyclohexan } \mathrm{H}-6) \\
4.31\left(\mathrm{~s}, 2 \mathrm{H}, \mathrm{NH}_{2}\right), 6.22 \\
(\mathrm{~s}, 1 \mathrm{H}, \mathrm{CH}=\mathrm{C}), 7.30-7.38 \\
\left(\mathrm{~m}, 5 \mathrm{H}, \mathrm{C}_{6} \mathrm{H}_{5}\right)\end{array}$ & $\begin{array}{l}23.6,25.3,37.9,44.2 \\
\text { (cyclohexyl C), 55.3, 84.2, } \\
95.2,101.1,11.7,113.0(3 \\
\mathrm{C}=\mathrm{C}), 116.1,116.8,117.2 \\
(3 \mathrm{CN}), 120.8,121.1,122.3 \\
124.7,126.0,128.1,130.2 \\
138.0 \text { (two benzene C) }\end{array}$ & 378 \\
\hline 8 & $\begin{array}{l}3420-3222(2 \mathrm{NH}), 3053 \\
(\mathrm{CH} \text { aromatic }), 2873 \\
\left(\mathrm{CH}_{2}\right), 2225,2220 \\
(2 \mathrm{CN}) 1655(\mathrm{C}=\mathrm{N}) \\
1630(\mathrm{C}=\mathrm{C})\end{array}$ & $\begin{array}{l}2.48-2.49\left(\mathrm{~m}, 4 \mathrm{H}, 2 \mathrm{CH}_{2}\right) \\
2.50-2.51\left(\mathrm{~m}, 4 \mathrm{H}, 2 \mathrm{CH}_{2}\right) \\
6.23(\mathrm{~s}, 1 \mathrm{H}, \mathrm{CH}=\mathrm{C}) \\
7.43-7.45\left(\mathrm{~m}, 10 \mathrm{H}, 2 \mathrm{C}_{6} \mathrm{H}_{5}\right) \\
8.23,8.45(2 \mathrm{~s}, 2 \mathrm{H}, 2 \mathrm{NH})\end{array}$ & $\begin{array}{l}24.2,25.8,35.8,43.8 \\
\text { (cyclohexyl C), 55.2, 80.6, } \\
94.0,111.2(2 \mathrm{C}=\mathrm{C}), 115.9, \\
116.3(2 \mathrm{CN}), 122.8,124.1, \\
126.3,129.0,131.8,132.1, \\
132.9,144.0(\text { two benzene } \\
\text { C). } 172(\mathrm{C}=\mathrm{N}), 180.3(\mathrm{C}=\mathrm{S})\end{array}$ & 435 \\
\hline
\end{tabular}


R. M. Mohareb and H. E. Moustafa: Use of 2-aminoprop-1-ene-1,1,3-tricarbonitrile for the synthesis of tetrahydronaphthalene, hexahydroisoquinoline and hexahydrocinnoline derivatives with potential antitumor activities, Acta Pharm. 61 (2011) 51-62.

\begin{tabular}{|c|c|c|c|c|}
\hline 9 & $\begin{array}{l}3465-3238\left(\mathrm{NH}_{2}, \mathrm{NH}\right) \\
3050(\mathrm{CH} \text { aromatic }) \\
2880\left(\mathrm{CH}_{2}\right), 2228 \\
2222,2220(3 \mathrm{CN}) 1659 \\
(\mathrm{C}=\mathrm{N}) 1635(\mathrm{C}=\mathrm{C})\end{array}$ & $\begin{array}{l}2.41-2.46\left(\mathrm{~m}, 4 \mathrm{H}, 2 \mathrm{CH}_{2}\right) \\
2.52-2.54\left(\mathrm{~m}, 4 \mathrm{H}, 2 \mathrm{CH}_{2}\right) \\
4.25\left(\mathrm{~s}, 2 \mathrm{H}, \mathrm{NH}_{2}\right), 7.32-7.40 \\
\left(\mathrm{~m}, 5 \mathrm{H}, \mathrm{C}_{6} \mathrm{H}_{5}\right), 8.09 \\
(\mathrm{~s}, 1 \mathrm{H}, \mathrm{NH})\end{array}$ & $\begin{array}{l}22.8\left(\mathrm{CH}_{3}\right), 24.6,26.2,35.3, \\
43.5(\text { cyclohexyl } \mathrm{C}), 55.0, \\
78.3,90.2,110.3(2 \mathrm{C}=\mathrm{C}), \\
115.8,116.2,116.9(3 \mathrm{CN}), \\
122.3,123.8,125.7,128.0 \\
\text { (benzene } \mathrm{C}), 176.3(\mathrm{C}=\mathrm{N})\end{array}$ & 330 \\
\hline 10 & $\begin{array}{l}3450-3322\left(\mathrm{NH}_{2}, \mathrm{NH}\right) \\
3055(\mathrm{CH} \text { aromatic }) \\
2980,2788\left(\mathrm{CH}_{3}, \mathrm{CH}_{2}\right) \\
2227-2222(2 \mathrm{CN}) 1655 \\
(\mathrm{C}=\mathrm{N}) 1640(\mathrm{C}=\mathrm{C}\end{array}$ & $\begin{array}{l}2.23-2.26\left(\mathrm{~m}, 4 \mathrm{H}, 2 \mathrm{CH}_{2}\right) \\
2.32-2.36\left(\mathrm{~m}, 4 \mathrm{H}, 2 \mathrm{CH}_{2}\right) \\
3.03\left(\mathrm{~s}, 3 \mathrm{H}, \mathrm{CH}_{3}\right), 5.44 \\
\left(\mathrm{~s}, 2 \mathrm{H}, \mathrm{NH}_{2}\right), 7.22-7.38(\mathrm{~m}, \\
\left.4 \mathrm{H}, \mathrm{C}_{6} \mathrm{H}_{4}\right), 8.25(\mathrm{~s}, 1 \mathrm{H}, \mathrm{NH})\end{array}$ & & 330 \\
\hline 11 & $\begin{array}{l}3463,3343\left(\mathrm{NH}_{2}\right) \\
3050(\mathrm{CH} \text { aromatic }) \\
2982,2884\left(\mathrm{CH}_{3}, \mathrm{CH}_{2}\right) \\
2229-2220(2 \mathrm{CN}) \\
1688(\mathrm{CO}), 1643(\mathrm{C}=\mathrm{N})\end{array}$ & $\begin{array}{l}2.24-2.26\left(\mathrm{~m}, 4 \mathrm{H}, 2 \mathrm{CH}_{2}\right), \\
2.35-2.38\left(\mathrm{~m}, 4 \mathrm{H}, 2 \mathrm{C}_{2}\right), \\
3.14\left(\mathrm{~s}, 3 \mathrm{H}, \mathrm{CH}_{3}\right), 4.68 \\
\left(\mathrm{~s}, 2 \mathrm{H}, \mathrm{NH}_{2}\right), 7.30-7.39 \\
\left(\mathrm{~m}, 4 \mathrm{H}, \mathrm{C}_{6} \mathrm{H}_{4}\right)\end{array}$ & & 331 \\
\hline $12 a$ & $\begin{array}{l}3448-3315\left(3 \mathrm{NH}_{2}\right) \\
3056(\mathrm{CH} \text { aromatic }) \\
2789\left(\mathrm{CH}_{2}\right), 2225 \\
(\mathrm{CN}), 1666(\mathrm{C}=\mathrm{N}) \\
1639(\mathrm{C}=\mathrm{C})\end{array}$ & $\begin{array}{l}2.21-2.26\left(\mathrm{~m}, 4 \mathrm{H}, 2 \mathrm{CH}_{2}\right), \\
2.30-2.38\left(\mathrm{~m}, 6 \mathrm{H}, 3 \mathrm{CH}_{2}\right), \\
4.21,5.20,5.24(3 \mathrm{~s}, 6 \mathrm{H}, \\
\left.3 \mathrm{NH}_{2}\right)\end{array}$ & & 244 \\
\hline $12 b$ & $\begin{array}{l}3470-3321\left(2 \mathrm{NH}_{2}, \mathrm{NH}\right) \\
3056(\mathrm{CH} \text { aromatic }) \\
2875\left(\mathrm{CH}_{2}\right) 1670 \\
(\text { exocyclic } \mathrm{C}=\mathrm{N}) \\
1638(\mathrm{C}=\mathrm{C})\end{array}$ & $\begin{array}{l}2.21-2.34\left(\mathrm{~m}, 10 \mathrm{H}, 5 \mathrm{CH}_{2}\right) \\
4.76,5.66\left(2 \mathrm{~s}, 4 \mathrm{H}, 2 \mathrm{NH}_{2}\right) \\
7.32-7.39\left(\mathrm{~m}, 5 \mathrm{H}, \mathrm{C}_{6} \mathrm{H}_{5}\right) \\
8.32(\mathrm{~s}, 1 \mathrm{H}, \mathrm{NH})\end{array}$ & $\begin{array}{l}24.8,25.9,36.0,43.3 \\
\text { (cyclohexyl C), 55.0, 78.3, } \\
90.2,110.3(2 \mathrm{C}=\mathrm{C}), 116.9 \\
(\mathrm{CN}), 120.2,122.5,123.4, \\
126.2,128.0 \text { (benzene C), } \\
169.2,172.8(2 \mathrm{C}=\mathrm{N})\end{array}$ & 320 \\
\hline
\end{tabular}

2-(Amino(2,3,5,6,7,8-hexahydro-3-imino-2-p-tolylcinnoline-4-yl)methylene)malononitrile (10). - A suspension of compound $9(0.33 \mathrm{~g}, 1 \mathrm{mmol})$ in sodium ethoxide solution (obtained by dissolving sodium metal $(0.046 \mathrm{~g}, 0.2 \mathrm{mmol})$ in absolute ethanol $(20 \mathrm{~mL})$ was heated in boiling water for $2 \mathrm{~h}$ and then left to cool. The solid product formed upon pouring onto ice/water was collected by filtration.

2-(Amino(3-oxo-2-(p-tolylamino)-2,3,5,6,7,8-hexahydrocinnolin-4-yl)methylene)malononitrile (11). - A solution of compound $10(0.330 \mathrm{~g}, 1 \mathrm{mmol})$ in ethanol $(30 \mathrm{~mL})$ containing one pellet of sodium hydroxide was heated under reflux for $3 \mathrm{~h}$, then poured onto ice/ water containing a few drops of hydrochloric acid and the formed solid product was collected by filtration.

3-Amino-3-(3,5-diamino-4H-pyrazol-4-ylidene)-2-cyclohexylidene-propanonitrile (12a) and 3-amino-3-(3-amino-5-imino-1-phenyl-1H-pyrazol-4- $(5 \mathrm{H})$-ylidene)-2-cyclohexylidenepropanonitrile (12b). - To a solution of compound $1(0.212 \mathrm{~g}, 1 \mathrm{mmol})$ in ethanol $(40 \mathrm{~mL})$, either hydrazine hydrate $(0.05 \mathrm{~g}, 1 \mathrm{mmol})$ or phenylhydrazine $(0.108 \mathrm{~g}, 1 \mathrm{mmol})$ was added. The reaction mixture in each case was heated under reflux for $3 \mathrm{~h}$ and then poured onto an ice/water mixture containing a few drops of hydrochloric acid. The solid product formed in each case was collected by filtration. 
R. M. Mohareb and H. E. Moustafa: Use of 2-aminoprop-1-ene-1,1,3-tricarbonitrile for the synthesis of tetrahydronaphthalene, hexahydroisoquinoline and hexahydrocinnoline derivatives with potential antitumor activities, Acta Pharm. 61 (2011) 51-62.

\section{Antitumor activity tests}

Fetal bovine serum (FBS) and L-glutamine were from Gibco Invitrogen Co. (UK). RPMI-1640 medium was from Cambrex (USA), dimethyl sulfoxide (DMSO), doxorubicin, penicillin, streptomycin and sulforhodamine B (SRB) were from Sigma Chemical Co. (USA).

Three human tumor cell lines, MCF-7 (breast adenocarcinoma), NCI-H460 (non-small cell lung cancer) and SF-268 (CNS cancer) were used. MCF-7 was obtained from the European Collection of Cell Cultures (ECACC, UK) and NCI-H460 and SF-268 were kindly provided by the National Cancer Institute (NCI, Cairo, Egypt). Their growth as a monolayer was routinely maintained in RPMI-1640 medium supplemented with $5 \%$ heat inactivated FBS, $2 \mathrm{mmol} \mathrm{L}^{-1}$ glutamine and antibiotics (penicillin $100 \mathrm{U} \mathrm{mL}^{-1}$, streptomycin $100 \mu \mathrm{g} \mathrm{mL}^{-1}$ ), at $37{ }^{\circ} \mathrm{C}$ in a humidified atmosphere containing $5 \% \mathrm{CO}_{2}$. Exponentially growing cells were obtained by plating $1.5 \times 10^{5}$ cells mL $\mathrm{mL}^{-1}$ for MCF-7 and SF-268 and $0.75 \times 10^{4}$ cells $\mathrm{mL}^{-1}$ for NCI-H460, followed by $24 \mathrm{~h}$ of incubation. The effect of the vehicle solvent (DMSO) on the growth of these cell lines was evaluated in all experiments by exposing untreated control cells to the maximum concentration $(0.5 \%)$ of DMSO used in each assay.

The effects of compound $\mathbf{2 - 1 2 b}$ on the in vitro growth of human tumor cell lines were evaluated according to the procedure adopted by the National Cancer Institute (NCI, USA) in the 'In vitro Anticancer Drug Discovery Screen' which uses the protein-binding dye sulforhodamine B to assess cell growth (12). Briefly, cells growing exponentially in 96-well plates were then exposed for $48 \mathrm{~h}$ to five serial concentrations of each compound, starting from a maximum concentration of $150 \mu \mathrm{mol} \mathrm{L}-1$. Following this exposure period, adherent cells were fixed, washed and stained. The bound stain was solubilized and the absorbance was measured at $492 \mathrm{~nm}$ in a plate reader (Powerwave XS, Bio-Tek Instruments Inc., USA). For each test compound and cell line, a dose-response curve was obtained and the growth inhibition of $50 \%\left(G_{50}\right)$, corresponding to the concentration of the compounds that inhibited $50 \%$ of net cell growth, was calculated as described elsewhere (13). Doxorubicin was used as a positive control and was tested in the same manner.

\section{RESULTS AND DISCUSSION}

\section{Chemistry}

The reaction of compound $\mathbf{1}$ with benzaldehyde gave the benzal derivative $\mathbf{2}$, the structure of which was established on the basis of analytical and spectral data. The ${ }^{1} \mathrm{H}$ NMR spectrum showed the presence of a multiplet at $\delta 2.22-2.24$ and 2.30-2.34 ppm coresponding to four $\mathrm{CH}_{2}$ groups, a singlet at $\delta 3.65 \mathrm{ppm}\left(\mathrm{D}_{2} \mathrm{O}\right.$ exchangeable corresponding to the $\mathrm{NH}_{2}$ group) and a singlet at $\delta 6.98 \mathrm{ppm}$ for the $\mathrm{CH}=\mathrm{C}$ proton. Moreover, the ${ }^{13} \mathrm{C}$ NMR spectrum showed the presence of signals at $\delta 26.8,27.3,29.0,30.3$ (cyclohexyl C), 116.0, 116.9, 117.3 (3 CN), 87.2, 90.5, 104.2, 106.2, 132.1, 140.3 (3 C=C), 124.1, 125.3, $128.8,129.5 \mathrm{ppm}$ (benzene C). The reactivity of the latter product with cyanomethylene reagents was studied. Thus, the reaction of compound 2 with either malononitrile or ethyl cyanoacetate gave the tetrahydronaphthalene derivatives $\mathbf{4 a}, \mathbf{b}$. The latter products 
were formed through intermediate formation of compounds $\mathbf{3} \mathbf{a}, \mathbf{b}$ (Scheme 1). Compounds $4 a$ and $4 b$ were obtained through another reaction route as well. Thus, the reaction of compound 1 with either $\alpha$-cyanocinnamonitrile or ethyl $\alpha$-cyanocinnamate gave the same tetrahydronaphthalene derivatives $4 \mathbf{a}$ and $4 \mathbf{b}$ (m.p. and mixed m.p.), respectively (Scheme 1). The analytical and spectral data (Tables I and II) of the latter products are consistent with the proposed structures (see experimental section).

On the other hand, the reaction of compound 2 with either hydrazine hydrate or phenylhydrazine gave the hyrazide derivatives $\mathbf{6} \mathbf{a}, \mathbf{b}$ through intermediate formation of $5 a, b$.

The reaction of compound 2 with bromine in glacial acetic acid gave the monobromo derivative 7 . The reaction of compound 2 with phenylisothiocynate gave the pyrimidine derivative $\mathbf{8}$. The analytical and spectral data of $\mathbf{7}$ and $\mathbf{8}$ (Tables I and II) are in agreement with the proposed structures (Scheme 2). The reaction of compound 1 with 4-methylbenzenediazonium chloride gave the hydrazone derivative 9 . The structure of compound 9 was also based on analytical and spectral data. Thus the ${ }^{1} \mathrm{H}$ NMR spectrum showed two multiplets at $\delta 2.22-2.28$ and $2.33-2.37 \mathrm{ppm}$ for four methylene groups, one

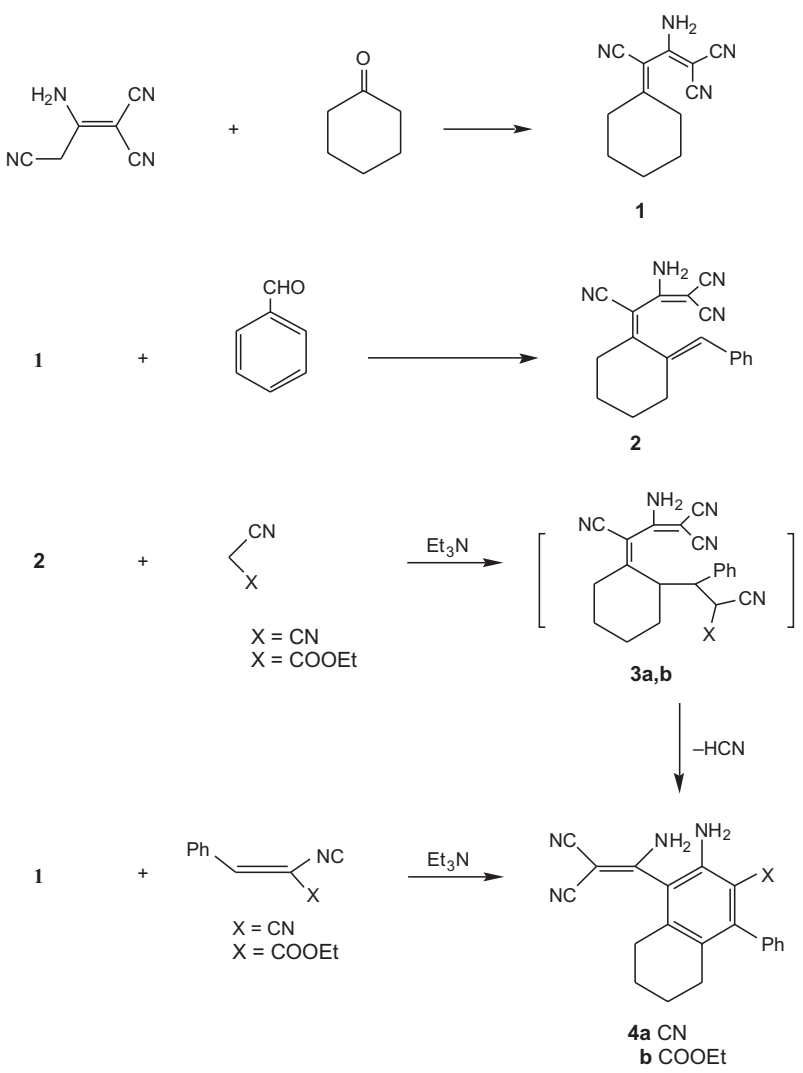

Scheme 1 


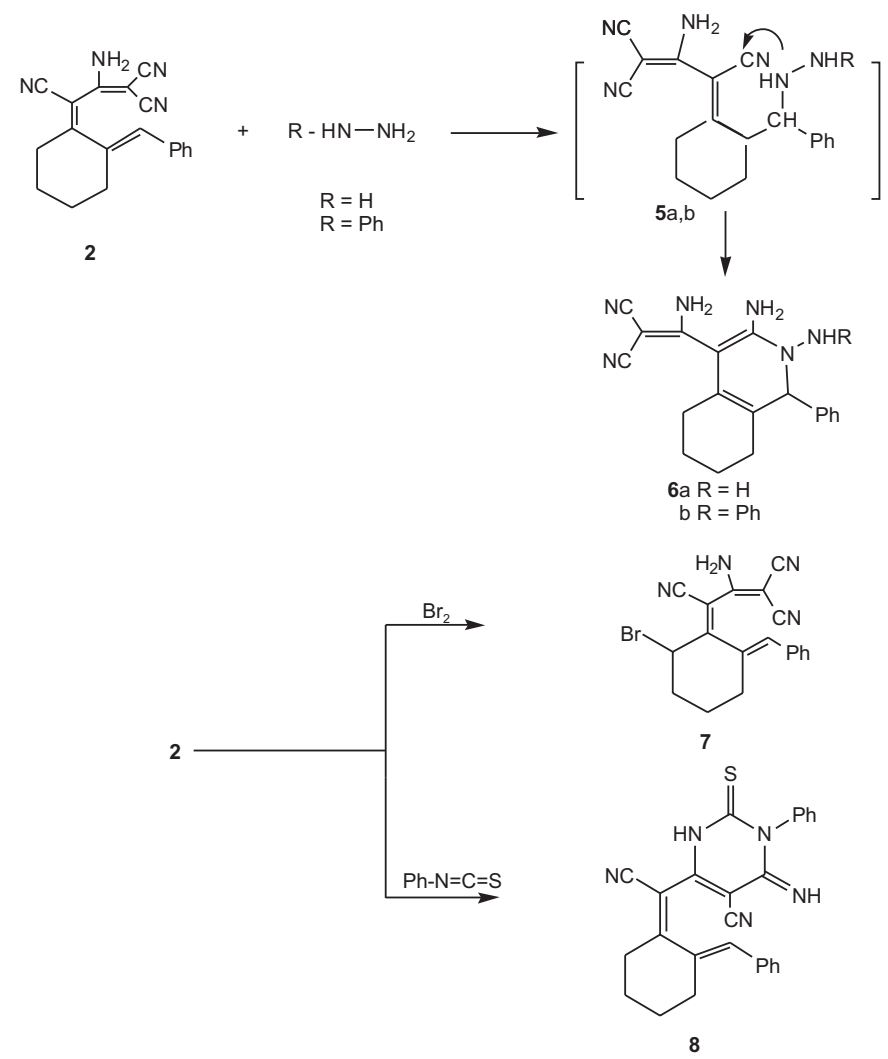

Scheme 2

singlet at $\delta 3.21$ ppm corresponding to $\mathrm{CH}_{3}$ group, and two singlets at $\delta 4.55$ and 8.88 ppm $\left(\mathrm{D}_{2} \mathrm{O}\right.$ exchangeable) corresponding to $\mathrm{NH}_{2}$ and $\mathrm{NH}$ groups. Moreover, ${ }^{13} \mathrm{C}$ NMR showed signals at $22.8\left(\mathrm{CH}_{3}\right), 24.6,26.2,35.3,43.5$ (cyclohexyl C), 55.0, 78.3, 90.2, 110.3 (2 $\mathrm{C}=\mathrm{C}), 115.8,116.2,116.9(3 \mathrm{CN}), 122.3,123.8,125.7,128.0$ (benzene $\mathrm{C}), 176.3$ ppm $(\mathrm{C}=\mathrm{N})$. Compound 9 underwent ready cyclization in sodium ethoxide solution to give the pyridazine derivative $\mathbf{1 0}$ (Scheme 3). The 6-imino group in compound $\mathbf{1 0}$ underwent ready hydrolysis when heated in ethanolic sodium hydroxide to give the 6-oxopyridazine derivative 11 (Scheme 3). Such hydrolysis of an imino group into an oxo group had been reported before $(14,15)$. The reaction of compound 2 with either hydrazine hydrate or phenylhydrazine gave the pyrazole derivatives 12a and $\mathbf{1 2 b}$, respectively (Scheme 3).

It is interesting to note that all compounds synthesized in this work can exist in either $Z$ or $E$ structures. However, according to the concept of push-pull alkenes reviewed by Sandstrom (16), conjugated systems with unsaturated heterocyclic rings with conjugated alkyl chains, exemplify typical push-pull compounds, which can exist in $Z / E$ equilibrium depending on the temperature and the nature of solvent. One can say that all the newly synthesized compounds in this work are typical push-pull compounds existing in $Z / E$ equilibrium. 


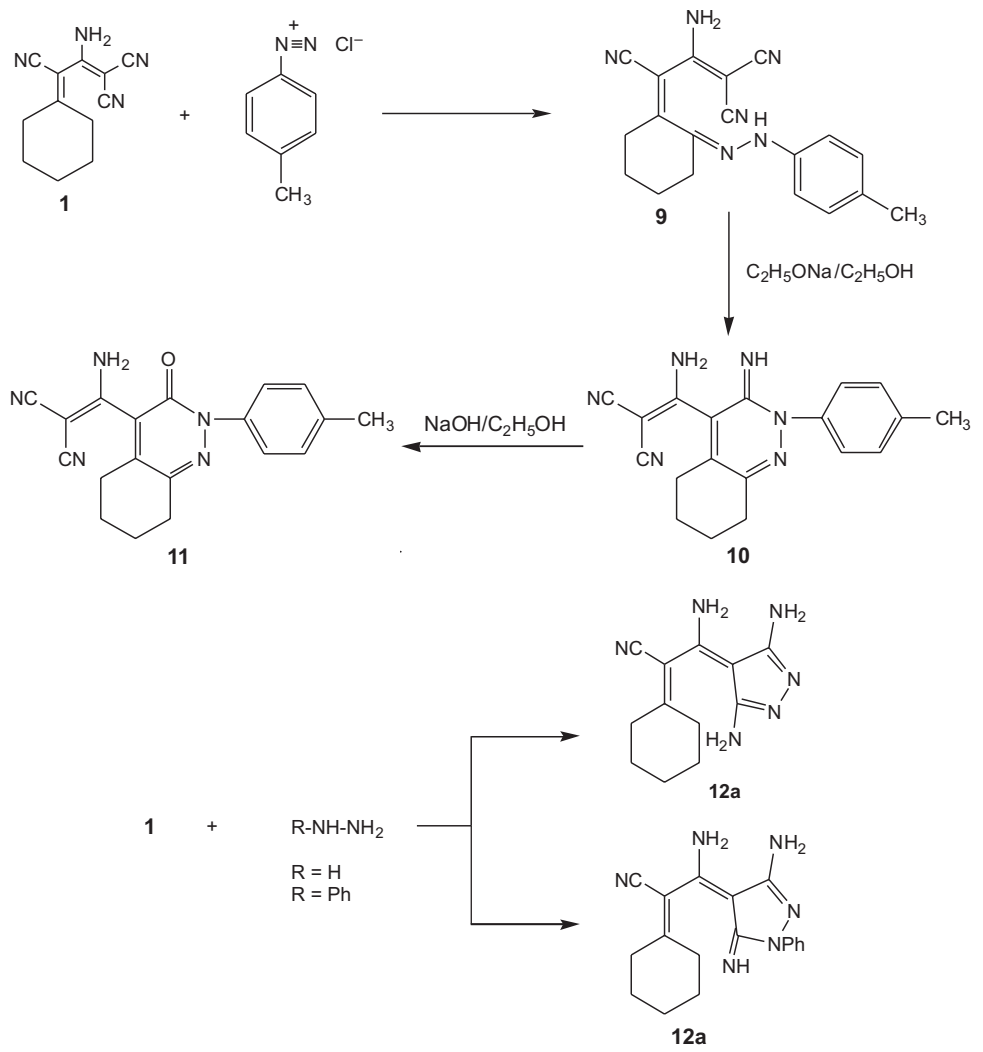

Scheme 3

\section{Antitumor activity}

The effect of compounds 1, 2, 4a, 4b, 6a, 6b, 7, 8, 9, 10, 11, 12a and $12 b$ was evaluated on the in vitro growth of three human tumor cell lines, namely, breast adenocarcinoma (MCF-7), non-small cell lung cancer (NCI-H460) and CNS cancer (SF-268), after continuous exposure for $48 \mathrm{~h}$. The results are summarized in Table III.

All compounds were able to inhibit the growth of the three human tumor cell lines in a dose-dependent manner (data not shown). The 2-(amino(3-phenylamino-1-phenyl-2-(phenylamino)-1,2,5,6,7,8-hexahydroisoquinolin-4-yl)methylene)malononitrile (6b) and the 3-amino-3-(3,5-diamino-4H-pyrazol-4-ylidene)-2-cyclohexylidene-propanenitrile (12a) exhibited the highest inhibitory effect on the three tumor cell lines, but still much lower than that of the control doxorubicin. On the other hand, compounds $4 a, 7$ and $\mathbf{1 2 b}$ showed a moderate growth inhibitory effect. Comparing the activities of $\mathbf{6} \mathbf{a}$ and $\mathbf{6} \mathbf{b}$, it was assumed that the N-phenyl group in $\mathbf{6 b}$ coused weaker growth inhibitory effect although the results in the NCI-H460 cell line were comparable. Moreover, it is convenient to compare the activity of 3-amino-3-(3,5-diamino- $4 \mathrm{H}$-pyrazol-4-ylidene)-2-cyclohexylide- 
R. M. Mohareb and H. E. Moustafa: Use of 2-aminoprop-1-ene-1,1,3-tricarbonitrile for the synthesis of tetrahydronaphthalene, hexahydroisoquinoline and hexahydrocinnoline derivatives with potential antitumor activities, Acta Pharm. 61 (2011) 51-62.

nepropanenitrile (12a) with that of 3-amino-3-(3-amino-5-imino-1-phenyl-1H-pyrazol-4-(5H)-ylidene)-2-cyclohexylidenepropanenitrile (12b): the former compound (with pyrazole moiety) showed higher inhibitory effect than the latter (with N-pyrazole moiety).

Table III. Effects of compounds 1-12b on the growth of three human tumor cell lines

\begin{tabular}{cccc}
\hline \multirow{2}{*}{ Compd. } & \multicolumn{3}{c}{$\mathrm{GI}_{50}(\mu \mathrm{mol} \mathrm{L}-1)$} \\
\cline { 2 - 4 } & MCF-7 & NCI-H460 & \multicolumn{1}{c}{ SF-268 } \\
\hline $\mathbf{1}$ & $30.1 \pm 0.6$ & $17.3 \pm 1.4$ & $22.3 \pm 1.5$ \\
$\mathbf{2}$ & $20.6 \pm 0.4$ & $24.3 \pm 0.8$ & $32 \pm 0.8$ \\
$\mathbf{4 a}$ & $70.6 \pm 16.9$ & $38.9 \pm 10.8$ & $50.8 \pm 8.6$ \\
$\mathbf{4 b}$ & $40.6 \pm 12.2$ & $32.6 \pm 8.6$ & $60.4 \pm 14.8$ \\
$\mathbf{6 a}$ & $35.4 \pm 10.2$ & $24.1 \pm 0.8$ & $18.9 \pm 6.8$ \\
$\mathbf{6 b}$ & $11.8 \pm 0.6$ & $14.5 \pm 0.8$ & $16.7 \pm 1.6$ \\
$\mathbf{7}$ & $72.7 \pm 17.5$ & $40.2 \pm 12.8$ & $50.0 \pm 9.0$ \\
$\mathbf{8}$ & $50.1 \pm 0.7$ & $23.2 \pm 4.8$ & $18.4 \pm 1.8$ \\
$\mathbf{9}$ & $22.0 \pm 0.2$ & $30.6 \pm 1.4$ & $38.4 \pm 0.6$ \\
$\mathbf{1 0}$ & $38.0 \pm 1.8$ & $44.0 \pm 0.8$ & $20.5 \pm 1.1$ \\
$\mathbf{1 1}$ & $20.0 \pm 0.6$ & $22.0 \pm 0.4$ & $31.5 \pm 8.0$ \\
$\mathbf{1 2 a}$ & $11.9 \pm 0.6$ & $14.1 \pm 0.6$ & $20.3 \pm 0.5$ \\
$\mathbf{1 2 b}$ & $70.9 \pm 0.9$ & $43.6 \pm 1.8$ & $56.8 \pm 0.8$ \\
Doxorubicin & $0.04 \pm 0.008$ & $0.09 \pm 0.008$ & $0.09 \pm 0.007$ \\
\hline
\end{tabular}

Results are given as concentrations that were able to cause $50 \%$ cell growth inhibition $\left(G I_{50}\right)$ after continuous exposure of $48 \mathrm{~h}$; Mean \pm SEM of three-independent experiments performed in duplicate.

\section{CONLUSIONS}

In this work, we described a new series of tetrahydronaphthalene, hexahydrophthalazine and hexahydrocinnoline derivatives obtained starting from the 2-amino-3-cyclohexylidene-1-propen-1,1,3-tricarbonitrile. Some of synthesized products showed potential anti-tumor activity. This finding encourages us to explore new molecules by introducing potent moieties, such as heterocyclic and fused ring systems described in this work, into other enaminonitriles. Our prediction is that these compounds with new ring systems may show better antitumor activity.

Acknowledgements. - R. M. Mohareb would like to thank the Alexander von Humboldt Foundation for a fellowship in Germany (Institute of Organic Chemistry, Friedrich-Alexander-University, Erlangen-Nürnberg) during the summer of 2009 and for completing the analytical data for this work. 


\section{REFERENCES}

1. A. Bolognes, G. Correale, M. Manfra, A. Lavecchia, E. Novellino and S. Pepe, Synthesis, structure-activity relationships, and biological evaluation of dimethyl-5H-pyridophenoxazin-5-ones, tetrahydro-5H-benzopyridophenoxazin-5-ones, and 5H-benzopyrido-phenoxazin-5-ones with potent antiproliferative activity, J. Med. Chem. 49 (2006) 5110-5118; DOI: 10.1021/jm0507451.

2. G. Heimer, M. R. Etizion, I. B. Gad, J. A. Goldberg, S. N. Haber and H. Bergman, Dopamine replacement therapy does not restore the full spectrum of normal pallidal activity in the 1-methyl-4-phenyl-1,2,3,6-tetra-hydropyridine primate model of Parkinsonism, J. Neurol. Sci. 6 (2006) 8101-8114; DOI: 10.1523/JNEUROSCI.5140-05.2006.

3. R. Powers, J. C. Copeland, K. Germer, K. A. Mercier, V. Ramanathan and P. Revesz, Comparison of protein active site structures for functional annotation of proteins and drug design, Proteins 65 (2006) 124-135; DOI: 10.1002/prot.21092.

4. L. K. Wing, H. A. Behanna, L. J. Van Eldik, D. M. Watterson and H. R. Ranaivo, De novo and molecular target-independent discovery of orally bioavailable lead compounds for neurological disorders, Curr. Alzheimer Res. 3 (2006) 205-214; DOI: 10.1016/S0163-7258(02)00297-8.

5. Z. Xin, M. D. Serby, H. Zhao, C. Kosogof, B. G. Szczepankiewicz, M. Liu, B. Liu, C. W. Hutchins, K. A. Sarris, E. D. Hoff, H. D. Falls, C. W. Lin, C. A. Ogiela, C. A. Collins, M. E. Brune, E. N. Bush, B. A. Droz, T. A. Fey, V. E. Knourek-Segel, R. Shapiro, P. B. Jacobson, D. W. Beno, T. M. Turner, H. L. Sham and G. Liu, Discovery and pharmacological evaluation of growth hormone secretagogue receptor antagonists, J. Med. Chem. 49 (2006) 4459-4469; DOI: 10.1021/jm060461g.

6. R. Sadzeviciene, J. Zekonis, G. Zekonis and P. Paipaliene, Oxidative functions of neutrophils in perioponditis patients with type 1 diabetes mellitus, Medicina (Kaunas) 42 (2006) 479-483.

7. R. Romagnoli, P. G. Baraldi, V. Remusat, M. D. Carrion, C. L. Cara, D. Petri, F. Fruttarolo, M. G. Pavani, M. A. Tabrizi, M. Tolomeo, S. Grimaudo, J. Balzarini, M. A. Jordan and E. Hamel, Synthesis and biological evaluation of 2-(3',4',5'-trimethoxy-benzoyl)-3-amino 5-aryl thiophenes as a new class of tubulin inhibitors, J. Med. Chem. 49 (2006) 6425-6428; DOI: 10.1021/jm060804a.

8. H. Zhao, M. D. Serby, Z. Xin, B. G. Szczepankiewicz, M. Liu, C. Kosogof, B. Liu, L. T. Nelson, E. F. Johnson, S. Wang, T. Pederson, R. J. Gum, J. E. Clampit, D. L. Haasch, C. Abad-Zapatero, E. H. Fry, C. Rondinone, J. M. Trevillyan, H. L. Sham and G. Liu, Discovery of potent, highly selective, and orally bioavailable pyridine carboxamide c-Jun $\mathrm{NH}_{2}$-terminal kinase inhibitors, J. Med. Chem. 49 (2006) 4455-4458; DOI: 10.1021/jm0604651.

9. J. M. Arif, M. Kunhi, A. A. Bekhit, M. P. Subramanian, K. Al-Hussein, H. Y. Aboul-Enein and F. M. Al-Khodairy, Evaluation of apoptosis-induction by newly synthesized phthalazine derivatives in breast cancer cell lines, Asian Pac. J. Cancer Prev. 7 (2006) 249-252; DOI: 10.1093/aje/ kwj264.

10. J. Fareh, R. M. Touyz, E. X. Achiffrin and G. Thibault, Altered cardiac endothelium receptors and protein kinase C in deoxycorticosterone-salt hypertensive rats, J. Mol. Cell. Cardiol. 32 (2000) 665-676; DOI: 10.1006/jmcc.2000.1110.

11. H. Junek, B. Thierrichter and P. Wibmer, Synthesis with nitriles, Comparative reactivity studies of the new codimers from malononitrile and ethyl cyanoacetate with carbonyl compounds, Monatsh. Chem. 110 (1979) 483-492.

12. P. Skehan, R. Storeng, D. Scudiero, A. Monks, J. McMahon, D. Vistica, J. T. Warren, H. Bokesch, S. Kenny and M. R. Boyd, New colorimetric cytotoxicity assay for anticancer-drug screening, J. Natl. Cancer Inst. 82 (1990) 1107-1112; DOI: 10.1093/jnci/82.13.1107.

13. A. Monks, D. Scudiero, P. Skehan, R. Shoemaker, K. Paul, D. Vistica, C. Hose, J. Langley, P. Cronise, A. Vaigro-Wolff, M. Gray-Goodrich, H. Campbell, J. Mayo and J. M. Boyd, Feasibility of a high-flux anticancer drug screen using a diverse panel of cultured human tumor cell lines, J. Natl. Cancer Inst. 83 (1991) 757-776; DOI: 10.1093/jnci/83.11.757. 
R. M. Mohareb and H. E. Moustafa: Use of 2-aminoprop-1-ene-1,1,3-tricarbonitrile for the synthesis of tetrahydronaphthalene, hexahydroisoquinoline and hexahydrocinnoline derivatives with potential antitumor activities, Acta Pharm. 61 (2011) 51-62.

14. R. M. Mohareb, S. S. Ghabrial and W. W. Wardakhan, The uses of 4-aryl-3-thiosemicarbazides in heterocyclic synthesis: synthesis of coumarin, pyrazole, thiazole and thiophene derivatives, Phosphorus Sulfur 134 (1998) 119-134; DOI: 10.1080/10426509808545457.

15. R. M. Mohareb and F. M. Manhi, Reaction of ethyl 2-diazo-4,5,6,7-tetrahydrobenzo[ $b]$ thiophene-3-carboxylate with 3-iminobutyronitrile: synthesis of pyridazines, thiophenes, and their fused derivatives, Heteroat. Chem. 11 (2000) 403-412; DOI: 10.1002/1098-1071.

16. J. T. Sandstrom, Measurement and calculation of [sup.13]C and [sup.15]N NMR chemical-shift tensors of a push-pull ethylene, Top. Stereochem. 14 (1983) 83-181; DOI: 10.1002/ 9780470147238.ch2.

$S A \check{Z} E T A K$

\section{Uporaba 2-aminoprop-1-en-1,1,3-trikarbonitrila u sintezi derivata tetrahidronaftalena, heksahidroizokinolina i heksahidrocinolina s potencijalnim antitumorskim djelovanjem}

RAFAT M. MOHAREB i HOSAM E. MOUSTAFA

Cilj rada bio je sintetizirati heterocikličke spojeve polazeći od 2-aminoprop-1-en-1,1,3-trikarbonitrila. Taj spoj je prvo u reakciji s cikloheksanonom dao derivat etilidena 2, iz kojeg su zatim priređeni derivati tetrahidronaftalena, heksahidrokinolina i heksahidrocinolina. Novosintetizirani spojevi ispitani su na antitumorsko djelovanje in vitro na tri humane tumorske stanične linije: adenokarcinoma grudi (MCF-7), tumora pluća (NCI-H460) i tumora CNS-a (SF-268). Neki od ispitanih spojeva pokazali su snažan inhibitorni učinak na ispitivane stanične linije, što ukazuje na mogućnost njihove primjene u razvoju onkoloških lijekova.

Ključne riječi: tetrahidronaftalen, heksahidrokinolin, heksahidrocinolin, antitumorsko djelovanje

October University for Modern Sciences and Arts, October City, A.R. Egypt

Chemistry Department, Faculty of Science, Cairo University, Giza, A.R. Egypt

Applied Medical Sciences Department, Community College in Quaryat, Al Jouf University, Al Jouf Saudia Arabia 